\title{
Substituição de sistema de retenção barra-clipe por Equator em overdenture sobre implante
}

\author{
Replacement of bar-clip retention system by Equator in overdenture on implant \\ Reemplazo del sistema de retención bar-clip por Equator en sobredentaduras sobre implantes
}

Recebido: 09/01/2021 | Revisado: 11/01/2021 | Aceito: 15/01/2021 | Publicado: 19/01/2021

Térsia Cristina Silva Macêdo

ORCID: https://orcid.org/0000-0002-1384-2202 Universidade Federal de Uberlândia, Brasil

E-mail: tersiiacristina@gmail.com

Camila de Carvalho Almança Lopes

ORCID: https://orcid.org/0000-0001-9313-9818 Universidade Federal de Uberlândia, Brasil

E-mail: milalopes_81@ hotmail.com

Fabiana Santos Gonçalves

ORCID: https://orcid.org/0000-0002-5560-1175 Curso Técnico em Prótese Dentária ESTES, Brasil

E-mail: fabi@ufu.br

Erick Rene Cerda-Rizo

ORCID: https://orcid.org/0000-0003-4316-1122

Universidad Americana, Nicaragua

E-mail: erickcerda8@gmail.com

Brenda Lisseth Pineda Mancia

ORCID: https://orcid.org/0000-0002-9243-381X

Universidade de Brasília, Brasil

E-mail: drapinedamancia@gmail.com

Morgana Guilherme de Castro Silverio

ORCID: https://orcid.org/0000-0002-0352-7946

Universidade Federal de Uberlândia, Brasil

E-mail: morgana.guilherme@ufu.br

\begin{abstract}
Resumo
A reabilitação oral de um paciente desdentado total requer atenção para sua correta avaliação, criterioso planejamento e realização adequada desse planejamento. $\mathrm{O}$ presente trabalho relata caso de substituição de sistema de retenção barra-clipe pelo sistema Equator de uma overdenture inferior. Paciente do sexo feminino, 70 anos de idade, compareceu ao projeto de extensão "Overdenture para desdentados totais na geriatria", queixando-se de um volume lingual grande da sua prótese inferior, dificultando sua alimentação e fonética e de fraturas sucessivas dessa prótese. $\mathrm{O}$ exame clínico intraoral revelou a presença de prótese total superior e overdenture inferior com o sistema de retenção barra-clipe, em que a posição espacial da barra estava inadequada e invadia o espaço da língua. Foi planejado a confecção de novas próteses e a substituição do sistema de retenção barra-clipe por um sistema independente. Ao levar o modelo de gesso inferior no delineador foi possível verificar que havia discrepância da inclinação dos implantes maior que $14^{\circ}$, selecionando-se assim o sistema Equator, o qual permite a captura de implantes com até $30^{\circ}$ de discrepância. Este caso evidencia a necessidade de um planejamento reverso para a realização das cirurgias de implantes, de forma que um correto sistema de retenção seja escolhido ainda durante planejamento e a etapa cirúrgica seja executada de maneira a atender esse planejamento. Após a colocação das novas próteses e do novo sistema de retenção a paciente relatou melhora significativa na alimentação e fonética, bem como da estética das próteses, gerando bem estar físico, psíquico e social.
\end{abstract}

Palavras-chave: Overdenture; Reabilitação; Implantes; Qualidade de vida.

\begin{abstract}
The oral rehabilitation of a total edentulous patient requires attention for its correct evaluation, careful planning and adequate planning. The present work reports a case of replacement of the bar-clip retention system by the Equator system of a lower overdenture. A 70-year-old female patient attended the extension project "Overdenture for total toothless in geriatrics", complaining about a large lingual volume of her lower prosthesis, making it difficult to eat and phonetics and successive fractures of that prosthesis. The intraoral clinical examination revealed the presence of an upper total prosthesis and a lower overdenture with the bar-clip retention system, in which the spatial position of the bar was inadequate and invaded the tongue space. It was planned to manufacture new prostheses and to replace the bar-clip retention system with independent systems. When taking the lower plaster model in the eyeliner, it was
\end{abstract}


possible to verify that there was a discrepancy in the inclination of the implants greater than $14^{\circ}$, thus selecting the Equator system, which allows the capture of implants with up to $30^{\circ}$ of discrepancy. This case highlights the need for reverse planning for the performance of implant surgery, so that a correct retention system is chosen during planning and the surgical stage is performed in order to meet this planning. After the placement of new prostheses and the new retention system, the patient reported a significant improvement in diet and phonetics, as well as in the aesthetics of the prostheses, generating physical, psychological and social well-being.

Keywords: Overdenture; Rehabilitation; Implants; Quality of life.

\section{Resumen}

La rehabilitación del paciente desdentado total requiere atención, evaluación correcta, planificación cuidadosa y planificación adecuada, promoviendo la salud física, psicológica y social. El documento informa el caso de la sustitución del sistema de sujeción barra-clipe por el sistema ecuador. Paciente de sexo femenino, de 70 años, que acudió al proyecto de extensión de sobredentadura para desdentado total en geriatría, con gran volumen lingual de prótesis inferior dificultades de alimentación, fonética, sucesivas fracturas de la prótesis. En el examen clínico, vimos una sobredentadura superior e inferior con el sistema de retención de barra-clip. Se encontró una posición espacial inadecuada de la barra, que invadió el espacio lingual, responsable de las fracturas de la base de la prótesis y de la dificultad en la alimentación y fonética. Se planificó fabricar nuevas prótesis y reemplazar el sistema de retención de barra-clip por sistemas independientes. Tomando los modelos de yeso con la captura de los implantes en el eyeliner, comprobamos que existía una discrepancia en la inclinación de los implantes superior a $14^{\circ}$, haciendo inviable el sistema O'ring. Elegimos el sistema ecuador que permite la captura de implantes con hasta $30^{\circ}$ de discrepancia. Este caso muestra la planificación inversa para realizar las cirugías de instalación de implantes para que se elija el sistema de retención correcto en la planificación y la cirugía se realice de acuerdo con la planificación. Luego de la instalación de nuevas prótesis y del nuevo sistema de retención, la paciente refirió mejoría en la dieta y fonética así como en la estética de sus prótesis, generando bienestar físico, psicológico y social.

Palabras clave: Sobredentadura; Rehabilitación; Implantes; Calidad de vida.

\section{Introdução}

A perda dentária leva a uma série de alterações nas estruturas bucais, envolvendo tecido ósseo, gengival, muscular e articulação temporo-mandibular (Assunção et al., 2004; Carvalho et al., 2018). Essas modificações acabam gerando uma diminuição da capacidade proprioceptiva, mastigatória (Taufer et al., 2016), fonética e estética (Sharma, Nagrath \& Lahori et al., 2017). Os pacientes edêntulos insatisfeitos com sua imagem, acabam por se ausentar dos círculos sociais (Barbieri, Rapoport et al., 2009; Carvalho et al, 2018). Assim sendo, devido ao aumento na expectativa de vida, mais do que a busca por restabelecer a função mastigatória que foi perdida, ao procurar o tratamento reabilitador, os pacientes buscam reconstituir sua imagem pessoal, social e seu bem-estar, obtendo uma melhora na sua qualidade de vida (Beloni, Vale \& Takahashi, 2013; Gonçalves et al., 2020; Silva et al., 2020).

A reabilitação de pacientes edêntulos, por meio de próteses totais, tem como principal objetivo restaurar a função mastigatória e fonética e devolver a aparência estética perdida, levando-os a um estado de bem estar físico, mental e social (Magalhães et al., 2006). Porém, há uma grande queixa por parte dos portadores de próteses totais inferiores quanto a sua retenção e estabilidade, uma vez que estes elementos básicos da biomecânica são dependentes das condições físicas e anatômicas do paciente (Dewan, Hems \& Owens, 2007).

Com o surgimento dos implantes osseointegrados, novas possibilidades e opções de tratamento surgiram (Veríssimo et al., 2021). Dentre elas, as overdentures sobre implantes, que atuam de forma semelhante à prótese total convencional, onde o suporte é predominantemente mucoso, porém a retenção e a estabilidade do aparelho são significantemente melhoradas pela da fixação aos implantes, se tornando uma prótese mucossuportada e implanto-retida (Fragoso et al., 2005).

Diferentes sistemas de retenção estão disponíveis no mercado e podem ser classificados em: barra-clipe, anel de retenção ou o'ring e magnetos (Telles et al., 2009). O sistema de retenção ideal para as overdentures deve proporcionar a prótese uma boa retentividade e estabilidade e deve ser de fácil manutenção. Para a eleição do melhor sistema, alguns critérios devem ser avaliados, dentre eles: o espaço protético intermaxilar, o rebordo alveolar, formato do rebordo, resiliência da 
mucosa, posicionamento dos implantes e paralelismo entre os implantes (Barcelos et al., 2014).

Um plano de tratamento minucioso deve ser conduzido levando em consideração pontos importantes como a anatomia maxilar e mandibular, o padrão de reabsorção óssea da região, a qualidade do osso disponível para a colocação dos implantes, o desenvolvimento do perfil de emergência e a função da maxila na fala e a estética (Bergamo et al., 2014). A qualidade e a quantidade óssea são fatores anatômicos que mais afetam a viabilidade e o prognóstico de qualquer reabilitação protética através de implantes dentários (Torcato et al., 2012). A melhor forma de avaliar esses pontos é realizar o planejamento reverso dessas reabilitações, reduzindo-se os imprevistos, procurando atingir o sucesso estético e funcional na reabilitação. Além de reduzir custos no tratamento e poder indicar qual o melhor sistema de retenção para as oververdentures (Amoroso, Andressa Paschoal et al., 2012).

Desta forma, o objetivo deste trabalho é apresentar um caso clínico de substituição de uma prótese total superior e de uma overdenture inferior retida por barra-clipe mal indicada, por novas próteses, mudando-se também o sistema de retenção da overdenture inferior.

\section{Metodologia}

Este trabalho refere-se a um relato de caso, descritivo e qualitativo. Pereira et al., (2018) discorrem que este tipo de trabalho se refere a uma descrição e análise o mais detalhada possível de algum caso que apresente alguma particularidade que o torna especial. A paciente procurou a Faculdade de Odontologia da Universidade Federal de Uberlândia para atendimento odontológico (Uberlândia/MG). Ela foi avaliada clinicamente, discutiu-se as possíveis opções de tratamento, e a paciente optou pela realização de novas próteses (total superior e overdenture inferior retida por sistema individual). Após os esclarecimentos de todas as vantagens e desvantagens do tratamento proposto, a paciente assinou o termo de consentimento livre e esclarecido.

\section{Relato de Caso}

Paciente do gênero feminino, 70 anos de idade compareceu ao projeto de extensão "Overdenture para desdentados totais na geriatria" na Escola Técnica de Saúde da Universidade Federal de Uberlândia, queixando-se de um volume lingual grande da sua prótese inferior que dificultava sua alimentação e fala, e com histórico de fraturas sucessivas da base dessa prótese. O exame clínico intraoral revelou a presença de uma prótese mucossuportada superior e uma overdenture inferior com o sistema de retenção do tipo barra-clipe (Figura 1-A). Ao remover as próteses de posição, verificou-se que a posição espacial da barra estava inadequada invadindo o espaço da língua (Figura 1-B). Consequentemente isso aumentava o volume lingual e gerava uma base lingual da prótese de espessura fina. Esse, provavelmente foi um dos fatores responsáveis pelas sucessivas fraturas da base da prótese e também pela dificuldade de alimentação e fala da paciente. Foi planejado a confecção de novas próteses totais superior e inferior, porém com a substituição do sistema de retenção barra-clipe por sistema independente, visto que os implantes estavam muito distantes entre si, posicionados próximo a região de canino. 
Figura 1. A) Próteses antigas sem caracterização e escultura adequadas; B) Posição inadequada da barra invadindo o espaço lingual.

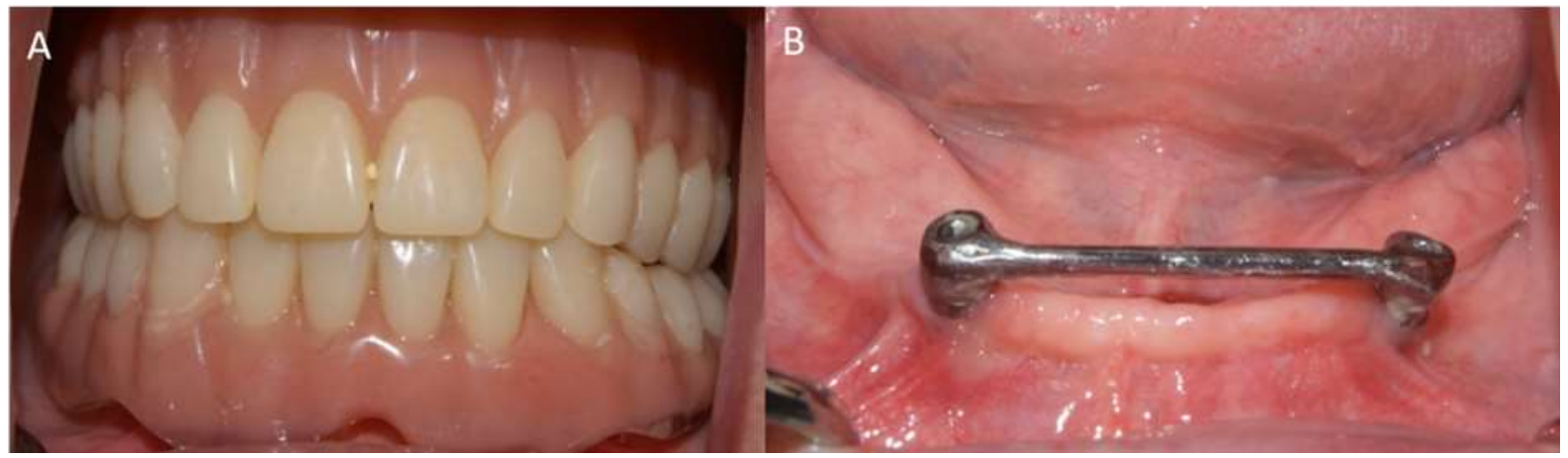

Fonte: Autores.

Foram realizadas moldagens anatômicas com moldeiras de estoque do tipo HDR para desdentados totais individualizadas com cera periférica (Lyzanda, São Paulo, SP, Brasil) e hidrocolóide irreversível (Jeltrate, Dentsply Sirona, São Paulo, SP, Brasil). Após a moldagem, os moldes foram lavados com água corrente e foi feita a desinfecção com hipoclorito de sódio 1\%. Em seguida foram confeccionados os modelos anatômicos em gesso pedra tipo III (Asfer Indústria Química, São Caetano do Sul, SP, Brasil) para confecção das moldeiras individuais. Após a delimitação da área chapeável e o alívio das áreas retentivas, bem como o alívio da região anterior inferior entre os implantes para a futura acomodação dos transferentes de moldagem dos implantes, as moldeiras individuais foram confeccionadas em resina acrílica ativada quimicamente (RAAQ). A moldagem funcional foi realizada em duas etapas, inicialmente foi feito o selado periférico com pasta zincoenólica (Lysanda), seguida da moldagem com elastômero tanto do arco superior quanto do arco inferior. No arco inferior a moldagem do rebordo foi feita juntamente com a transferência dos implantes. Após desinfecção dos moldes com hipoclorito $1 \%$, foram confeccionados os modelos de trabalho com gesso pedra tipo IV (Durone, Dentsply).

O modelo de trabalho inferior foi levado ao delineador e foi possível visualizar que havia grande discrepância da inclinação dos implantes entre si (Figura 2), o que inviabilizava a escolha do sistema O'ring, pois com este sistema não seria possível obter um eixo de inserção único para a prótese. A indicação desse sistema é para implantes paralelos entre si ou com discrepâncias de no máximo $14^{\circ}$. Foi então escolhido o sistema Equator (Neodent, Curitiba, PR, Brasil) que permite a captura de implantes com até $30^{\circ}$ de discrepância entre eles, de acordo com o fabricante.

Figura 2 A e B: Modelo de gesso levado ao delineador, foi verificado que os implantes estavam divergentes entre si.

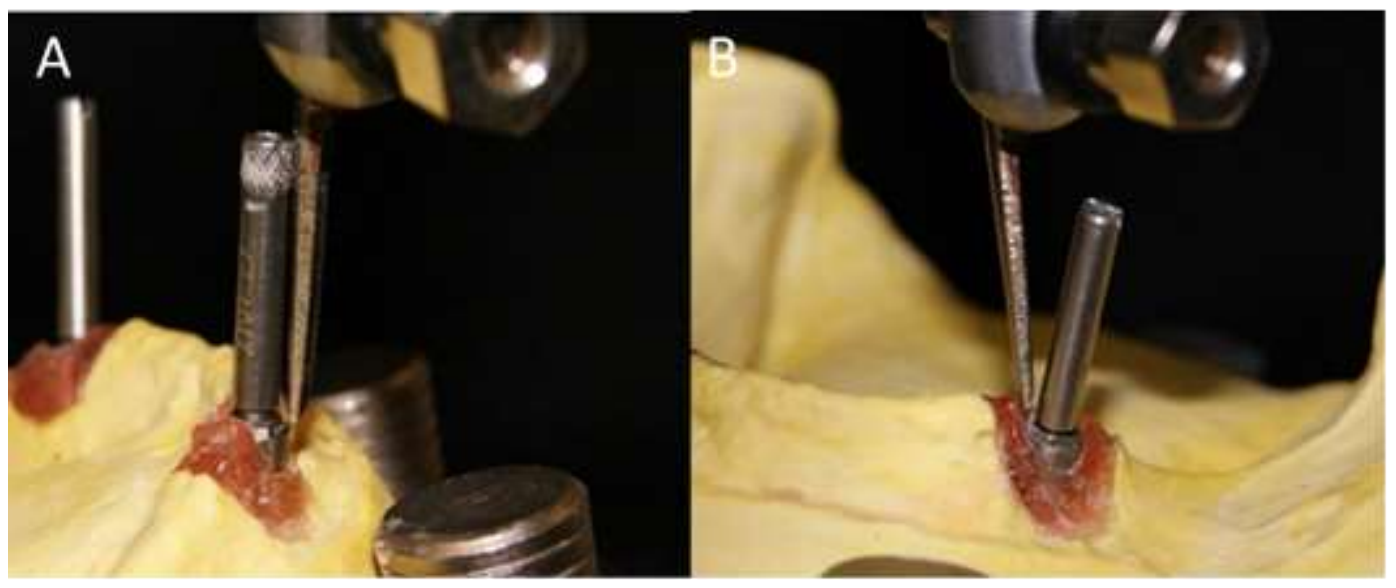

Fonte: Autores. 
Sobre os modelos de trabalho foram confeccionadas as bases de prova e plano de cera para a realização do registro maxilomandibular. O registro maxilomandibular foi executado de maneira a conter as seguintes informações: suporte de lábio, altura incisal, inclinação do plano oclusal, corredor bucal, linhas de referência para seleção e montagem dos dentes artificiais, dimensão vertical de oclusão e registro da relação cêntrica. Em seguida foi realizada a montagem dos modelos em articulador semi- ajustável (ASA, Bioart, São Paulo, SP, Brasil). A seleção de dentes seguiu as seguintes características: formato do rosto, características físicas, formato do rebordo alveolar, gênero e idade. A seleção da cor da gengiva foi realizada utilizando a escala do Sistema Tomas Gomes (STG, São Paulo, SP, Brasil). Foi realizada a montagem dos dentes e o enceramento caracterizado (Figura 3), pois a caracterização nessa etapa diminui o contraste da cera com o dente, diminuindo a atenção que o paciente deposita na cera vermelha, bem como se assemelha a prótese final e assim, durante a prova funcional de dentes o paciente tem uma ideia mais próxima de como ficará a sua futura prótese quando acrilizada. Durante a prova funcional e estética dos dentes foram avaliadas todas as características determinadas durante o registro intermaxilar (Figura 4).

Figuras 3. Montagem de dentes e enceramento caracterizado.

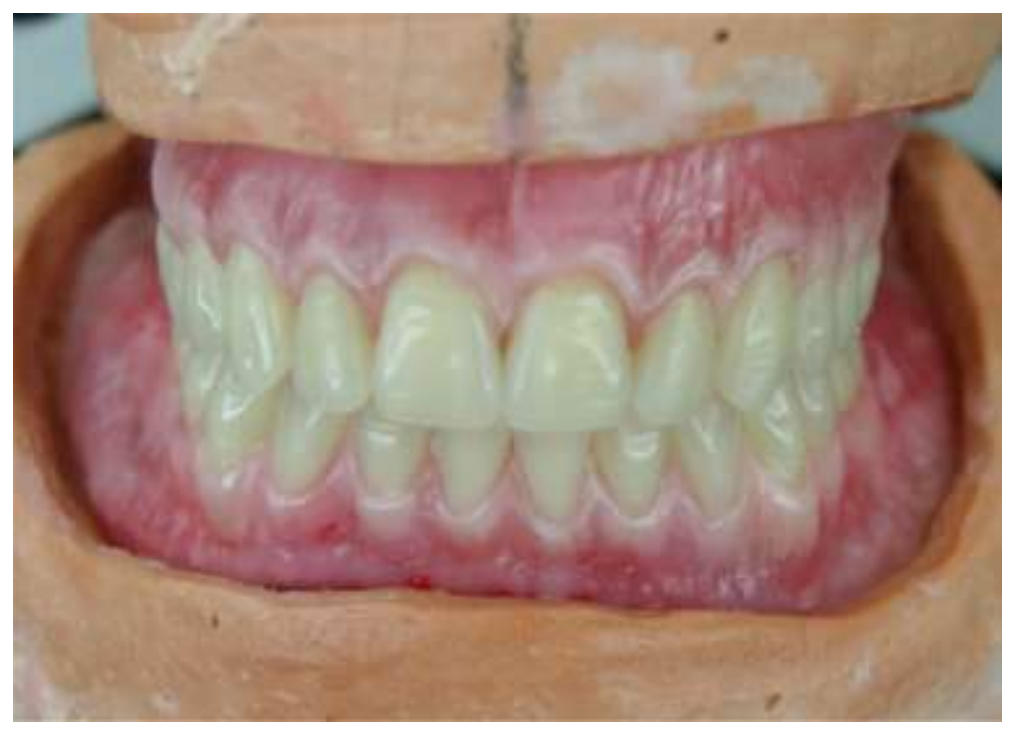

Fonte: Autores.

Figura 4. Prova funcional e estética dos dentes. A) Vista frontal; B) Vista lateral.

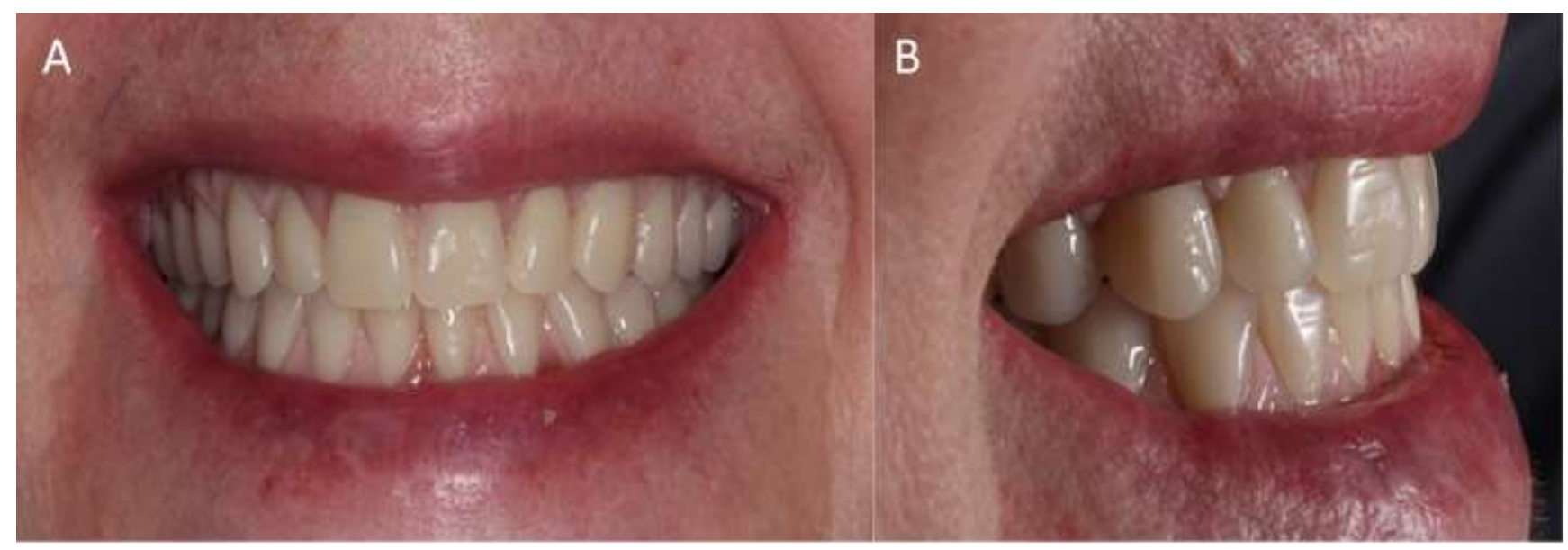

Fonte: Autores. 
Após a prova funcional dos dentes, passou-se para o processamento laboratorial das bases da prótese que compreendeu: inclusão em mufla, eliminação da cera, caracterização da gengiva com o sistema STG, polimerização convencional (ciclo australiano), demuflagem, acabamento e polimento com brocas maxicut de corte cruzado fino, lixa d'água e torno elétrico. Ao final do processo, obteve-se próteses bem polidas e acabadas (Figura 5).

Figura 5. Próteses totais acrilizadas.

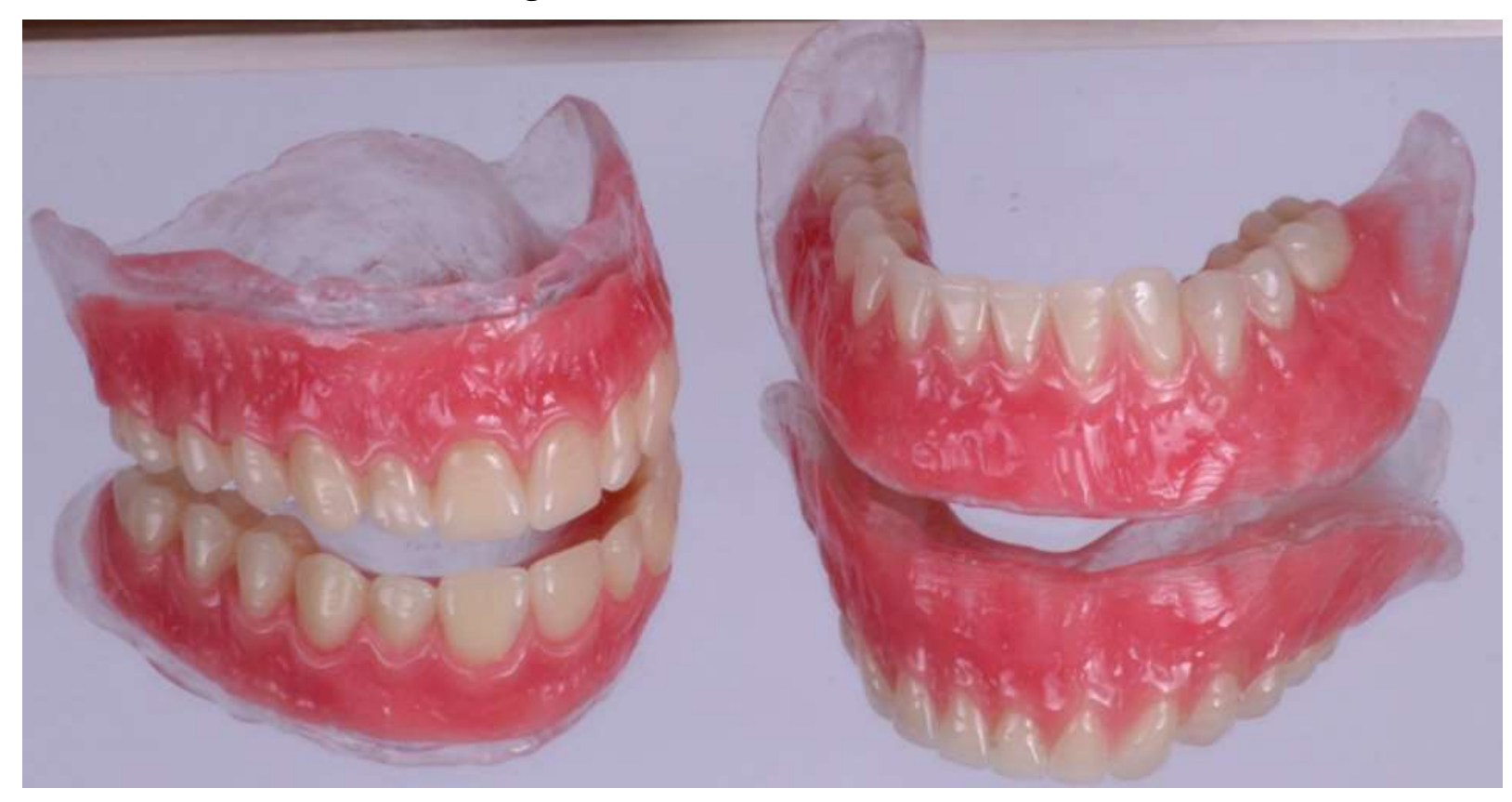

Fonte: Autores.

As próteses foram instaladas sem a captura imediata. A paciente as utilizou por 30 dias para acomodação dos tecidos, nesse período foram feitos os controles posteriores para os ajustes necessários. Quando a paciente relatou que as próteses não mais machucavam e estavam estáveis e confortáveis, procedeu-se a captura. Os pilares Equator foram instalados com 32N de torque, seguindo a recomendação do fabricante (Figura 6-A). Em seguida procedeu-se a colocação dos discos de proteção para captura e das cápsulas metálicas com inclinações diferentes de maneira a garantir um eixo de inserção único para a prótese, visto que os implantes estavam divergentes entre si (Figura 6-B). 
Research, Society and Development, v. 10, n. 1, e38710111829, 2021

(CC BY 4.0) | ISSN 2525-3409 | DOI: http://dx.doi.org/10.33448/rsd-v10i1.11829

Figura 6. A) Instalação do pilar Equator. B) Cápsulas em posição prontas para a captura.

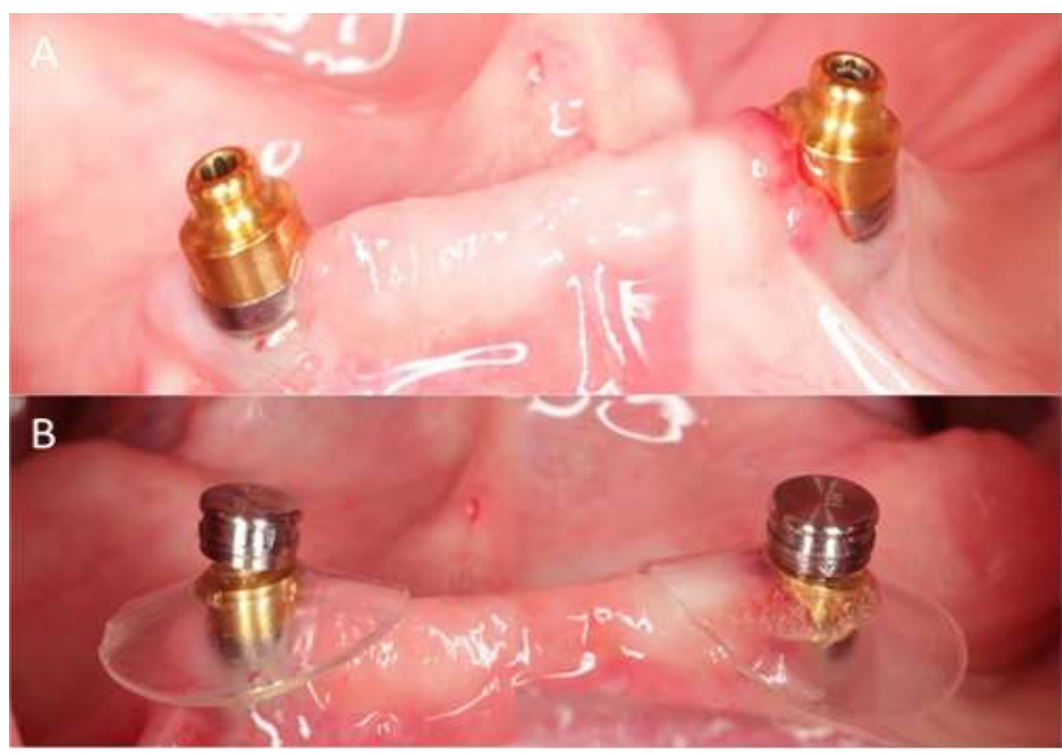

Fonte: Autores.

Foi realizado o alívio com broca maxicut de corte cruzado fino na prótese na região onde seriam capturadas as cápsulas metálicas (Figura 7-A). A captura foi realizada colocando RAAQ tanto no alívio na base da prótese como sobre as cápsulas metálicas e levando a prótese em posição em boca, com o paciente em oclusão. Após a polimerização da RAAQ, retirou-se a prótese da boca da paciente e procedeu-se o preenchimento de eventuais espaços vazios entre as cápsulas e a base da prótese. Findada a etapa de captura, foi feito o acabamento e polimento da RAAQ da captura (Figura 7-B). Após a colocação das novas próteses e do novo sistema de retenção, a paciente relatou melhora significativa da sua alimentação e fala, bem como da estética de suas próteses gerando efetivo bem estar físico, psíquico e social nessa paciente (Figura 8).

Figura 7. A) Alívio da prótese inferior com maxicut na região das cápsulas para captura da prótese; B) Captura da prótese inferior.

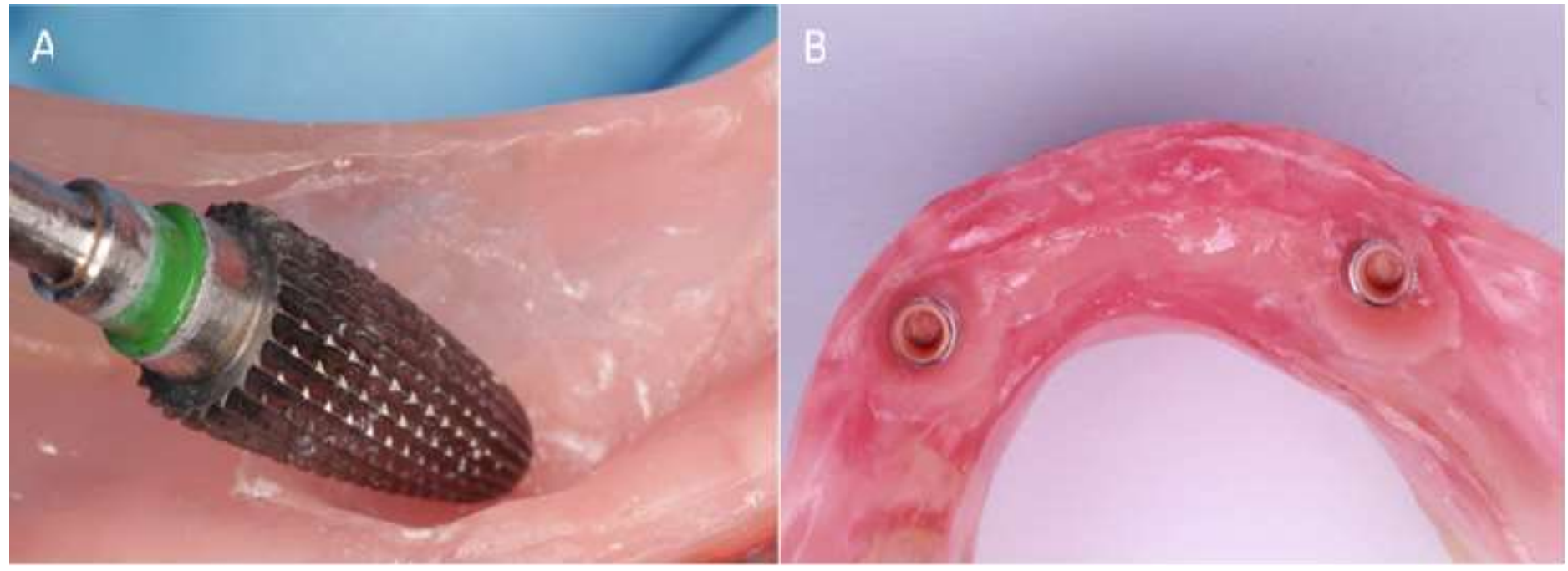

Fonte: Autores. 
Figura 8. Resultado final das próteses instaladas e da overdenture inferior capturada.

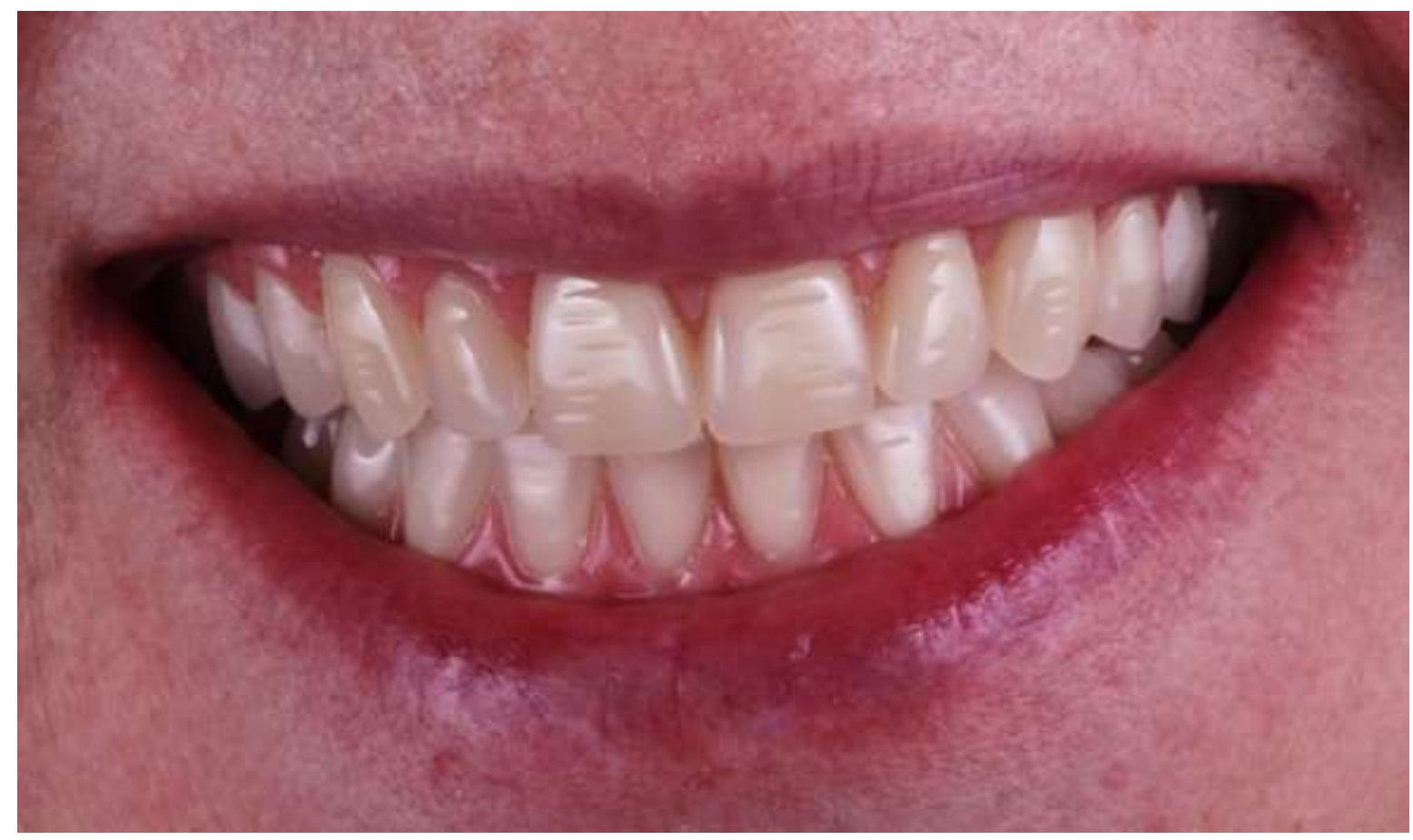

Fonte: Autores.

\section{Discussão}

Um grande número de pacientes portadores de prótese total convencional inferior relata insatisfação quanto a sua pouca retenção e estabilidade, o que acaba diminuindo assim a sua capacidade mastigatória, autoconfiança, auto-estima e convívio social (Novaes, Seixas \& Goiato, 2008). Com isso, as overdentures implantossuportadas e próteses tipo protocolo são frequentemente indicadas em mandíbulas edêntulas, pois elas oferecem uma melhor retenção e estabilidade (Johns et al., 1992).

Uma das vantagens da overdenture quando comparada à prótese do tipo protocolo, é seu custo. Em casos de reabilitações mandibulares são necessários dois implantes ao invés de quatro, normalmente indicados para as próteses do tipo protocolo. Desta forma, há uma menor necessidade de procedimentos cirúrgicos adicionais, como por exemplo, enxertos ósseos (Winkler, Morris \& Ochi, 2000). A quantidade de sessões clínicas e etapas laboratoriais também é menor, gerando menor custo (Assad et al., 2004.). Além disso, elas são de fácil higienização quando comparadas as próteses do tipo protocolo, pois é possível remover a prótese para realizar a escovação e limpeza dos attachments e da prótese em si (Novaes et al., 2008).

O sistema barra-clipe exibido pela paciente na primeira consulta, apresenta-se como um sistema de encaixe de um clipe a uma barra, que une 2 ou mais implantes (Chung et al., 2004). Ele apresenta ótima retenção e há uma melhor distribuição das cargas aos implantes devido à esplintagem pela barra (Rocha et al., 2020). Como grande limitação, o sistema precisa de um espaço mínimo de $14 \mathrm{~mm}$ entre a plataforma do implante e a borda incisal do dente da prótese (Tabata et al., 2007). Este espaço é necessário para posicionar a barra a $2 \mathrm{~mm}$ de altura em relação ao rebordo alveolar, para que se possa ter uma higienização correta e o não desenvolvimento de hiperplasias. Outros 5,5 mm são necessários para acomodar os componentes do sistema, e os milimetros restantes refere-se à altura dos dentes artificiais. Precisa-se ter o cuidado também de manter uma espessura mínima da base da prótese de $2 \mathrm{~mm}$ (Phillips e Wong et al., 2001), evitando fratura da prótese na região do desgaste para acomodação do clipe. Além disso, deve-se atentar ao comprimento e posicionamento da barra no momento da sua indicação. Para evitar que a barra sofra deflexão durante sua função, é necessário que seu comprimento seja de no máximo 
de $20 \mathrm{~mm}$ (Misch et al., 2005) e no mínimo de 12mm (Trakas et al., 2006). Seu posicionamento espacial deve ser de tal forma que não invada o espaço da língua, nem fique excessivamente para vestibular dos implantes, pois isso acaba dificultando o posicionamento dos dentes artificiais e sua higienização (Telles et al., 2009).

O posicionamento incorreto da barra pode gerar algumas complicações que poderiam ser evitadas com um planejamento reverso. Segundo Cardoso et al. 2005, a posição mais favorável para se instalar os implantes deve ser decidida e avaliada no pré-operatório, além da escolha do melhor sistema de retenção para o caso. Por isso, durante o exame clínico, caso o paciente já utilize próteses totais, devemos avaliá-las e se estiverem satisfatórias e com uma DVO correta, deve-se usá-las no planejamento reverso. Caso o paciente não faça o uso de próteses ou estas estiverem com uma DVO inadequada, deve-se realizar montagem em articulador dos modelos, com uma correta relação maxilo-mandibular para visualizar o espaço intermaxilar que está disponível, para assim definirmos o melhor sistema de retenção e o melhor posicionamento dos implantes. Quando o paciente apresentar reduzida reabsorção óssea anterior na maxila e/ou mandíbula, ou quando os implantes são instalados imediatamente após a extração dentária, pode-se ter dificuldades em indicar um tratamento reabilitador com overdenture pela falta de espaço intermaxilar. Nestes casos, se uma prótese do tipo overdenture foi escolhida como opção de tratamento, torna-se necessário durante a cirurgia, a remoção óssea gerando espaço suficiente para o sistema de retenção e da futura prótese (Telles et al., 2009).

Os sistemas de anel de retenção ou O'ring, são compostos por um intermediário tipo encaixe esférico ou anelar e um anel de náilon alojado em um estojo ou cápsula metálica que fica presa à base de resina acrílica da prótese total (Telles et al., 2009). Nos sistemas de anel de retenção, o paralelismo entre os implantes é muito importante e deve ser muito bem avaliado, pois não se deve ter uma divergência maior que $14^{\circ}$ entre eles. Caso haja uma divergência maior que esta, o paciente terá dificuldade na inserção e remoção da prótese overdenture, e isso levara há um maior desgaste dos anéis de retenção, comprometendo também a distribuição das cargas mastigatórias entre osso/implante (Spiekermann et al., 2000). Após a moldagem funcional da paciente, o modelo de trabalho inferior foi levado ao delineador, sendo possível visualizar discrepância da inclinação dos implantes, maior que $14^{\circ}$.

Entretanto, alguns sistemas individuais de anel de retenção oferecem componentes para correção da divergência entre os implantes, o que nos permite adquirir um paralelismo do eixo de inserção da prótese, seja pelo uso de componentes angulados ou pela forma de captura (Telles et al., 2009). Isto torna esses sistemas bastante versáteis, pois são capazes de solucionarem esse tipo de situação clínica (Telles et al., 2009). Podemos citar o sistema ERA®, sistema Locator (Straumann), e o sistema Equator (Neodent, Curitiba, Paraná, Brasil). O sistema Equator, eleito para a utilização no caso clínico relatado, além de possuir uma cápsula com pouca altura, também permite corrigir uma angulação de até $30^{\circ}$ de divergência entre dois implantes (Telles et al., 2009).

\section{Conclusão}

Com a substituição do sistema de retenção barra-clipe pelo sistema Equator, obteve-se uma prótese sem a invasão do espaço da língua, refletindo no conforto da paciente ao conversar e se alimentar. Outro benefício relatado foi maior facilidade de higienização dos attachments, por se tratar de sistemas individuais. Dessa forma, a solução do caso foi satisfatória, devolvendo à paciente bem estar físico, psíquico e social. Este caso também evidencia a necessidade de se utilizar um planejamento reverso para que o correto sistema de retenção seja previamente escolhido e a etapa cirúrgica seja executada de maneira a atender esse planejamento. 


\section{Agradecimentos}

Aos alunos e funcionários do Curso de Prótese dentária da Escola Técnica de Saúde (ESTES/UFU) pelo auxílio nas etapas laboratoriais de confecção das próteses totais do caso clínico.

\section{Referências}

Amoroso, A. P., Gennari Filho, H., Pellizzer, E. P. Goiato, M. C. Santiago Júnior, J. F., \& Villa, L. M. R. (2012). Planejamento reverso em implantodontia: relato de caso clínico. Rev. odontológica de araç, 33(2), 75-79.

Assad, A. S., Abd El-Dayem, M. A., \& Badawy, M. M. (2004). Comparação entre as sobredentaduras mandibulares suportadas principalmente por mucosa e combinadas por implantes. Implant dent, 13(4), 386-94.

Assunção, W. G. (2004). Anatomia para-protética: importância em prótese total. Rev Odontol Arac, 25(1), 57-62.

Barbieri, C. H., \& Rapoport, A. (2009). Avaliação da qualidade de vida dos pacientes reabilitados com próteses implanto-muco-suportadas versus próteses totais convenientes. Rev bras círculo da cabeça, 38(2), 84-87.

Barcelos, B. A. (2014). Avaliação da satisfação e qualidade de vida relacionada à saúde bucal de pacientes usuários de prótese overdenture mandibular retida por um único implante (Tese de doutorado). Universidade Federal de Goiás, Goiânia, GO, Brasil.

Batista, A. V. D., Castro, P. E. O., Batista, J. D., Arioli Filho, N., \& Carvalho, F. A. A. (2006). Overdenture retira por barra: uma alternativa à prótese total. ROBRAC, 15(40), 78-84.

Beloni, W. B., Vale, H. F., Takahashi, J. M. F. K. (2013). Avaliação do grau de satisfação e qualidade de vida dos portadores de prótese dental. RFO, 18(2), $160-164$.

Bergamo, E. T. P., Barbi, F. C. L., Farahr, G. J., Celestrino, M., Pereira, J. R., \& de Melo M. P. (2014). Reabilitação de maxila edêntula com prótese fixa implantossuportada: relato de caso. Dental Press Implantology, 8(4), 16-26.

Cardoso, A. C. (2005). O passo a passo da prótese sobre implante. Santos.

Carvalho, L. F., Melo, J. R. O., Ramos, J. G., Lima, R. A., \& Carvalho, F. A. A. (2019). Anatomia para-protética: o impacto do edentulismo na qualidade de vida de pacientes edentulosência em prótese total. Revista da acbo, 8(1), 40-48.

Chung, K. H., Chung, C. Y., Cagna JR, D. R., \& Cronin, R. J. (2004). Características de retenção de sistemas de fixação para overdentures de implante. J prosthodont, 13(4), 221-6.

Dewan, K., Hems, E., \& Owens, J. (2007). Um estudo retrospectivo para avaliar a satisfação do paciente com próteses estabilizadas por implante, 34(8), 470477.

Fragoso, W. S., Henriques, G., Mesquita, M., Nóbilo, M., Júnior, M. G., \& Oliveira, L. V. (2004). Reabilitação estética e funcional com sobredentadura implanto-retida: relato de caso. Odonto. clín.-cient., 3(3), 217-225.

Gonçalves, F. P., Alves, G., Oliveira, F., Antunes, L. A. A., Soares, J. R. A., Perazzo, M. F., Paiva, S. M., \& Scelza, M. F. Z. (2020). Impacto da reabilitação oral na qualidade de vida e nos níveis de cortisol de pacientes geriátricos. Research, Society and Development, 9(11), e2639119911. https://doi.org/10.33448/rsd-v9i11.9911

Johns, R. B., Jemt, T., Heath, M. R., Hutton, J. E., McKenna, S., McNamara, D. C., van Steenberghe, D., Taylor, R., Watson, R. M., \& Herrmann, I. (1992). A multicenter study of overdentures supported by Brånemark implants. The International journal of oral \& maxillofacial implants, 7(4), 513-522.

Magalhães, A. C. P., \& Gennari Filho, H. (2006). Distúrbios articulares nos desdentados totais. Rev. Ciênc. Ramal, 2(2), 1-16.

Misch, C. E. (2005). Prótese sobre implantes. In: Desenho e confecção da overdenture sobre implantes na mandíbula. Santos.

Novaes, L. C. G. F., Seixas, Z. A., \& Goiato, M. C. (2008). Prótese total sobre implante: técnicas contemporâneas e satisfação do paciente. Int J Dent, 7(1), $50-62$.

Pereira, A. S., Shitsuka, D. M., Parreira, F. J., \& Shitsuka, R. (2018). Metodologia do trabalho científico. Ed. UAB/NTE/UFSM. https://repositorio. ufsm. br/bitstream/handle/1/15824/Lic_Computacao_Metodologia-Pesquisa-Cientifica. pdf.

Philips, K., \& Wong, K. M. (2001). Requisitos de espaço para overdentures de barra e clipe retidos por implantes. Compend contin educ dent, 22(6), 516-518.

Sharma, A. J., Nagrath, R., \& Lahori, M. (2017). Uma avaliação comparativa da eficiência da mastigação, força de mordida mastigatória e satisfação do paciente entre dentadura convencional e sobredentadura mandibular suportada por implante: Um estudo in vivo. J Indian Prosthodont Soc, 17(4), 361-372.

Silva, R. A. R., Almeida Neto, L. F., Marcelino, K. P., Cardoso, L. C. L., Dantas, E. M., Barbosa, G. A. S., Macêdo, F. C., \& Gondim, A. L. M. F. (2020). Segmental osteotomy of the maxilla in the aesthetic area to enable rehabilitation with dental implants: case report. Research, Society and Development, 9(9), e594997525. https://doi.org/10.33448/rsd-v9i9.7525

Rocha, Sicknan Soares da. (2020). Reabilitação Oral Prótese Sobre Implante Na Era Digital. Quintessence.

Spiekermann, H. (1995). Implantologia. Atlas colorido de odontologia. Artmed. 
Research, Society and Development, v. 10, n. 1, e38710111829, 2021 (CC BY 4.0) | ISSN 2525-3409 | DOI: http://dx.doi.org/10.33448/rsd-v10i1.11829

Tabata, L. F., Assunção, W. G., Rocha, E. P., Zuim, P. R. J., \& Gennari Filho, H. (2007). Critérios para seleção dos sistemas de retenção para overdentures implanto-retidas. $R V O, 12(1), 75-80$.

Telles, D. (2009). Prótese Total-convencional e sobre implantes. Santos.

Torcato, L. B., Pellizzer, E. P., \& Goiato, M. C. (2012). Aspectos clínicos influentes no planejamento das próteses sobre implantes tipo overdenture e protocolo. Rev. Odontol de Arac., 33(2), 52-58.

Trakas, T., Michalakis, K., Kang, K., \& Hirayama, H. (2006). Sistemas de fixação para sobredentadura retida por implante: uma revisão da literatura. Implant dent, 15(1), 24-34.

Veríssimo, A. H., Souza, J A. N., Oliveira, T. A., Gonçalves, A. G., Afonso, F. A. C., \& Souza Júnior, F. A. (2021). Reabilitação oral com implante dentário e carga imediata por cirurgia guiada: relato de caso. Pesquisa, Sociedade e Desenvolvimento, 10 (1), e4810110854. https://doi.org/10.33448/rsd-v10i1.10854

Winkler, S., Morris, H. F., Ochi, S. (2000). Sobrevida do implante até 36 meses em relação ao comprimento e diâmetro. Annals periodontology, dec, 5(1), 2231. 